\title{
Management of Home Parenteral Nutrition: A Prospective Multicenter Observational Study
}

\author{
Carla Aeberhard $^{a} \quad$ Michèle Leuenberger $^{a} \quad$ Maya Joray $^{a}$ Peter E. Ballmer ${ }^{b}$ \\ Stefan Mühlebach ${ }^{c}$ Zeno Stanga ${ }^{a}$ \\ aDepartment of Endocrinology, Diabetes and Clinical Nutrition, Bern University Hospital and University of Bern, \\ Bern, ${ }^{b}$ Department of Medicine, Kantonsspital Winterthur, Winterthur, ' ${ }^{\circ}$ Department of Clinical Pharmacy and \\ Epidemiology and Hospital Pharmacy, University of Basel, Basel, Switzerland
}

\section{Key Words}

Home parenteral nutrition - Patient characteristics .

Underlying disease $\cdot$ Quality of life $\cdot$ Complications

\begin{abstract}
Background: There are no specific Swiss home parenteral nutrition (HPN) data showing patient characteristics, quality of life (QoL) and complications. The goal of this study was to collect representative nationwide data on current adult HPN patients in Switzerland for international comparability and benchmarking. Methods: This was a multicenter, nationwide, observational study. We conducted interviews for demographics, PN characteristics, QoL and complications. The data were assessed at baseline and after a follow-up of 3 months using a questionnaire. Results: Thirty-three adult patients were included. The most common underlying diseases were cancer, radiation enteritis and state after bariatric surgery, and the most prevalent indication was short bowel syndrome. During the 3-month observation period, significant increase or stabilization of body weight occurred in the patients, physical activity scores improved from 34.0 to 39.4 and mental scores improved from 41.9 to 46.4 . HPN dependency and traveling restrictions were of the greatest concern. Diarrhea, xerostomia and/or thirst were frequent com-
\end{abstract}

plaints. Conclusion: Anthropometric parameters and QoL improved during the observational period in this HPN cohort. These Swiss HPN data are prerequisite for evaluation and comparison of HPN recommendations and best clinical practice, status of professional care instructions related to HPN effectiveness, quality of treatment and patient safety.

(c) 2015 S. Karger AG, Basel

\section{Introduction}

Parenteral nutrition (PN) is life saving and indicated when the gut is not functioning and due to oral or enteral intake not being sufficient to reach appropriate requirement target. Advances in home parenteral nutrition (HPN) enable many patients to survive, to live at home and to have good or reasonably good quality of life (QoL) in their normal environment instead of prolonged hospital stays. Today it represents an established and commonly used procedure [1]. Even in elderly patients with cancer, HPN has been shown to benefit nutritional status and QoL [2]. Patients can be treated in an outpatient setting, resulting in reduced healthcare costs [3].

Since the introduction of HPN in the 1970s, HPN prevalence and incidence have been steadily increasing in

\section{KARGER 125}

(c) 2015 S. Karger AG, Base

0250-6807/15/0674-0210\$39.50/0

E-Mail karger@karger.com

www.karger.com/anm
Carla Aeberhard, Pharmacist, PhD Student

Department of Endocrinology, Diabetes and Clinical Nutrition

Bern University Hospital and University of Bern

Freiburgstrasse 4, CH-3010 Bern (Switzerland)

E-Mail carla.aeberhard@insel.ch 
Europe, with a large multicentric survey showing an annual incidence of 4-6 per $10^{6}$ inhabitants and a prevalence of 2-40 per $10^{6}$ inhabitants [4-6]. The most common indications for long-term HPN in Europe are Crohn's disease, mesenteric vascular disease, cancer and radiation enteritis [7]. Other more recent studies confirm that malignancy is the single most common indication for HPN $[8,9]$.

PN causes important restrictions in personal life, particularly, in social life, mainly due to the cyclic nocturnal HPN administration. These patients show reduced QoL, with physical problems, HPN dependency, social restrictions, sleeping problems and financial problems [10].

Shaw et al. [8] performed a first retrospective epidemiological analysis of patients with home artificial nutrition in Switzerland, based on data provided by the leading national insurance provider. Over the 5-year analysis period, from 2005 to 2009, $433 \mathrm{HPN}$ patients were recorded, showing increasing frequency of HPN.

Data on HPN patients' characteristics and living conditions are scarce, and little is known about the challenges and problems these patients face. The goal of this prospective study was to collect representative nationwide data on the current Swiss adult HPN patient group for international comparability and benchmarking.

\section{Material and Methods}

\section{Study Design and Patient Selection}

This multicenter, nationwide, observational study started in April 2013 and ended in March 2014. General practitioners and hospital physicians taking care of HPN patients were contacted. Their names were accessible through the Swiss Association for Common Tasks of Health Insurers (SVK). The physicians addressed were requested to obtain written consent from their patients to participate in the study. Inclusion criteria were the following: age $>18$ years, receiving HPN, life expectancy $>30$ days and signed written consent. Patients fulfilling the inclusion criteria were either visited at home for an interview or interviewed in their hospital's outpatient clinic.

\section{Data Collection}

Data were collected using an entry questionnaire and a followup questionnaire 3 months later. Patients included in the study either filled out the questionnaire together with their interviewer or on their own. The mean interview duration was about $45 \mathrm{~min}$. The following data were collected: personal and demographic data, social aspects, detailed nutrition regimes and QoL according to the validated Short Form 36 Health Survey version 2 (SF-36v2) ${ }^{\mathrm{TM}}$ questionnaire, anthropometric data over time, indications for $\mathrm{PN}$, medical history and prevalence of complications. The documents were available in three languages: German, French and Italian.
Body mass index (BMI) was calculated as weight in kilograms divided by height in meters squared $\left(\mathrm{kg} / \mathrm{m}^{2}\right)$. To assess the effects of HPN on personal life, behavior and emotions, patients were interviewed about the effects of HPN and asked to check all applicable responses. HPN-related complaints could be evaluated by the patients in the questionnaire by choosing from 3 options: ' $\mathrm{a}$ little', 'medium' or 'strong'. To assess physical performance and mental health status at different time points (before disease, just before starting HPN and during HPN), the patients could choose between 4 different options ('very good', 'good', 'bad' and 'very bad'). The QoL of the patients on HPN was assessed using the SF$36 \mathrm{v} 2^{\mathrm{TM}}$ questionnaire at the time of entry in the study and at the time point when the patients had to answer the follow-up questionnaires.

The SF-36v2 ${ }^{\mathrm{TM}}$ is a multipurpose health survey with 36 questions. It yields an 8-scale profile of functional health and wellbeing scores as well as psychometrically based physical and mental health summary measures and a preference-based health utility index [11]. These 8 scales are reduced to a physical component summary $(\mathrm{PCS}=$ physical functioning + physical role + bodily pain + general health) and to a mental component summary $(\mathrm{MCS}=$ vitality + social functioning + emotional role + mental health) [12].

\section{Statistical Analysis}

The statistical assessment was performed with IBM SPSS statistics for Windows, version 19.0 (IBM Corp., Released 2010, Armonk, N.Y., USA). The Wilcoxon test was used for continuous, non-parametric data. Results are reported as means with SD or as numbers and percentages. A p value of $<0.05$ was considered statistically significant.

\section{Ethical Approval}

This study was conducted in accordance with the ethical guidelines of the 1957 Declaration of Helsinki, and written informed consent was obtained from all participants. Ethical approval for the study was obtained from the Bernese Cantonal Ethics committee (KEK Bern, study no. 068/13), Bern, Switzerland.

\section{Results}

Forty-one patients were recorded during the 1-year investigation (fig. 1). Eight of the patients who were comparable to the study population did not consent to participate, while 33 participated $(n=100 \%)$ and filled in the entry questionnaire. The follow-up questionnaire was filled in by 24 patients. In 3 patients, the loss of follow-up was because they died during the study period due to the underlying disease (cancer), 4 patients ceased HPN and 2 patients had no more interest in the further participation of the study. This corresponds to about 4 patients per 1 million inhabitants in Switzerland and is comparable with other European countries. All tertiary university hospitals and all important referral centers of different regions of Switzerland, as well as namely physicians, who 


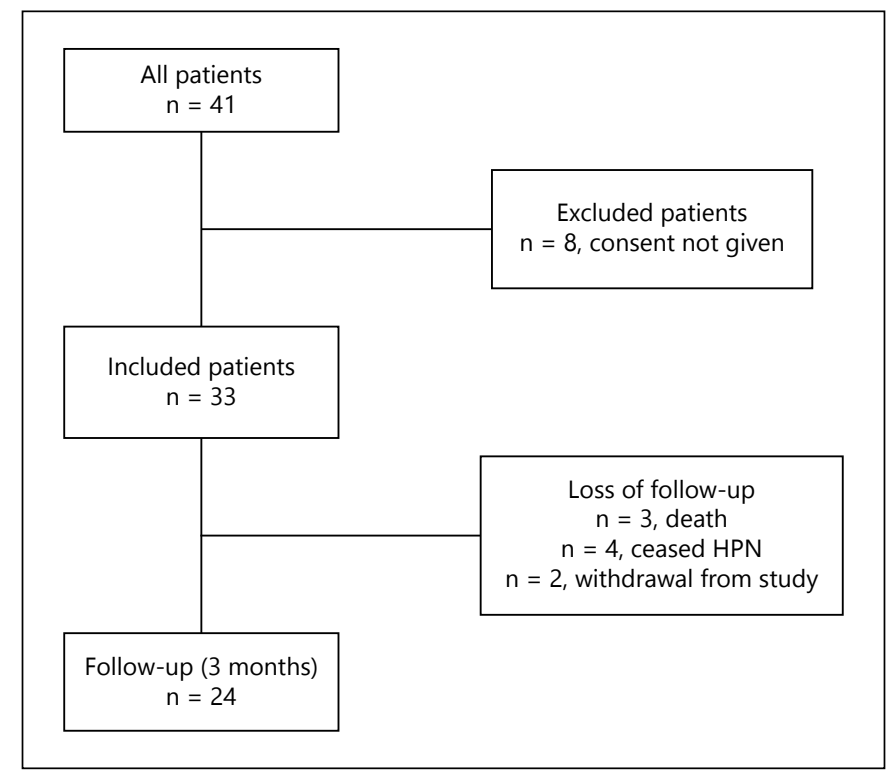

Fig. 1. Study flow diagram.

Table 1. Patient characteristics

\begin{tabular}{|c|c|}
\hline Data & Results \\
\hline \multicolumn{2}{|l|}{ Patients, gender, n (\%) } \\
\hline Male & $12(36)$ \\
\hline Female & $21(64)$ \\
\hline \multicolumn{2}{|l|}{ Age at start of HPN, years } \\
\hline Age, mean $\pm \mathrm{SD}$ & $53.76 \pm 17.75$ \\
\hline Age, median & 59 \\
\hline \multicolumn{2}{|l|}{ Age, n (\%), (f:m) } \\
\hline$<40$ & $8(24),(5: 3)$ \\
\hline $41-60$ & $11(33),(9: 2)$ \\
\hline $61-70$ & $10(30),(5: 5)$ \\
\hline$\geq 71$ & $4(12),(2: 2)$ \\
\hline \multicolumn{2}{|l|}{ Geographical distribution (Switzerland), $\mathrm{n}(\%)$} \\
\hline East part & $1(3)$ \\
\hline North part & $2(6)$ \\
\hline South part & $3(9)$ \\
\hline Central part & $5(15)$ \\
\hline West part & $10(30)$ \\
\hline Region of Berne & $12(36)$ \\
\hline \multicolumn{2}{|l|}{ Marital status } \\
\hline Married & $15(45)$ \\
\hline Single & $9(27)$ \\
\hline Divorced & $7(21)$ \\
\hline Widowed & $2(6)$ \\
\hline \multicolumn{2}{|l|}{ Living situation, $\mathrm{n}(\%)$} \\
\hline Partner & $15(45)$ \\
\hline With children $<18$ years or with the parents & $3(9)$ \\
\hline Alone & $8(24)$ \\
\hline Nursing home & $4(12)$ \\
\hline \multicolumn{2}{|l|}{ Catheter types, n (\%) } \\
\hline Hickman & $18(55)$ \\
\hline Port-a-cath & $14(42)$ \\
\hline Dialysis fistula & $1(3)$ \\
\hline
\end{tabular}

are familiar with HPN therapies, were contacted but not all of them had patients to contribute. The patients' characteristics are listed in table 1 . The mean age on starting HPN was $53.8 \pm 17.8$ years. At the time of the initial interview, HPN treatment had been initiated on an average of 3.44 years previously, with a wide range from 2 days to 30.75 years. The mean BMI before disease was $28.5 \pm 11.4$ $\mathrm{kg} / \mathrm{m}^{2}$ and decreased significantly before starting HPN to a BMI value of $19.6 \pm 6.7 \mathrm{~kg} / \mathrm{m}^{2}(\mathrm{p}<0.001)$. At the time point of the first interview, the mean BMI had increased significantly with HPN treatment to $22.0 \pm 6.1 \mathrm{~kg} / \mathrm{m}^{2}(\mathrm{p}<$ 0.001 ). The BMI could be kept stable at $21.5 \pm 3.2$ until the follow-up interview (fig. 2).

\section{Underlying Diseases and Indications for HPN}

The underlying diseases and the indications for HPN of the 33 patients are reported in table 2 . As underlying disease cancer was predominant $(\mathrm{n}=14,42 \%)$, followed by post-bariatric surgery and post-radiation enteritis $(\mathrm{n}=$ 4 each, 12\%). In 13 (39\%) of the cases, a short bowel syndrome (SBS) was the indication for HPN.

\section{Central Venous Catheter-Related Complications}

Most patients ( $\mathrm{n}=32,97 \%)$ used tunneled catheter systems like the Hickman or the Port-a-cath. Only 1 patient was fed through a dialysis arteriovenous fistula. In $45 \%$ of the patients $(n=15)$, there was at least one central venous catheter (CVC) replacement during the nutritional treatment, primarily due to infections, CVC occlusions or displacement. Eleven patients (33\%) reported at least one infection during the HPN period. Interventionrelated CVC complications (e.g. arterial lesions, fixation problems) occurred in 5 patients (15\%) and CVC occlusions in 5 patients (15\%). During the present observational period (follow-up questionnaire after 3 months from baseline), 6 patients (18\%) had to have the CVC replaced due to infectious complications.

\section{Effects of HPN on QoL}

Regarding the employment, $85 \%(\mathrm{n}=28)$ of the HPN patients were no longer employed, either because of physical impairment or because they had already retired ( $\mathrm{n}=$ $13)$. Nine percent $(n=3)$ had an employment with medium physical activity or were students. Less than half of the patients $(39 \%, \mathrm{n}=13)$ were able to do the housekeeping themselves; $58 \%(n=19)$ were dependent on family help. Personal care was performed themselves by $88 \%$ $(\mathrm{n}=29)$. For attaching and removing the PN, 76\% ( $\mathrm{n}=$ 25) relied on external help, such as a homecare service or a nurse. 
Fig. 2. The time course of BMI (mean \pm SD).

Fig. 3. Activity and mental health followup.
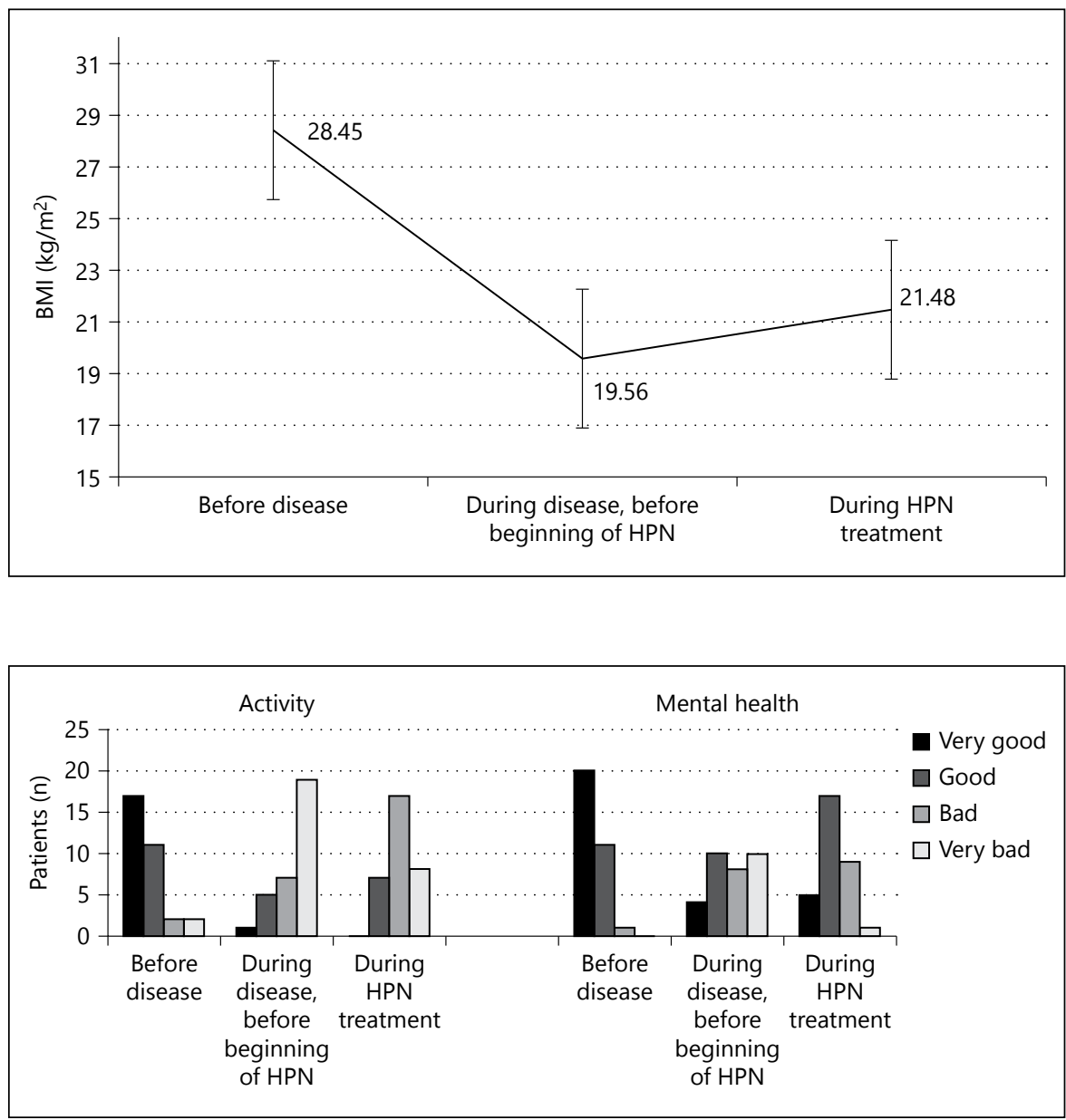

Performance level and mental health status are shown in figure 3. Before the disease, $52 \%(n=17)$ of patients were very active. During the disease, but just before beginning HPN, $58 \%(n=19)$ were not active at all. This improved during treatment with HPN, with $52 \%(\mathrm{n}=17)$ of patients reporting they were a little active. The mental health status was rated very bad in $30 \%(\mathrm{n}=10)$ during the disease, just before starting HPN. During HPN treatment, only $3 \%(n=1)$ described their mental status as very bad. Almost all patients showed improved physical and mental QoL after 3 months (PCS 34.02 vs. 39.37; MCS 41.91 vs. 46.35), as shown in figure 4 . Only 2 patients (6\%) considered themselves healthy, while 7 (21\%) described themselves as fairly healthy. Most patients reported in the QoL questionnaire that their health was not excellent at all $(\mathrm{n}=12,36 \%)$.

Aspects of HPN treatment that were reported as most disturbing were the restricted ability to travel and the impossibility to participate in social events $(\mathrm{n}=21,64 \%)$ and the dependency on the treatment $(n=19,58 \%)$. Sleep
Table 2. Underlying diseases and indication for HPN

\begin{tabular}{|c|c|}
\hline Diagnosis & $\mathrm{n}(\%)$ \\
\hline Cancer & $14(42)$ \\
\hline Complications of surgery & $5(15)$ \\
\hline Post-bariatric & $4(12)$ \\
\hline Others & $1(3)$ \\
\hline Radiation enteritis & $4(12)$ \\
\hline Crohn's disease & $3(9)$ \\
\hline Systemic sclerosis & $2(6)$ \\
\hline Congenital bowel disease (Hirschsprung disease) & $1(3)$ \\
\hline Motility disorders & $1(3)$ \\
\hline Caecum perforation & $1(3)$ \\
\hline Mesenteric infarction & $1(3)$ \\
\hline Glycogenosis type Ia ( $->$ severe inappetence) & $1(3)$ \\
\hline Indication for HPN & $\mathrm{n}(\%)$ \\
\hline SBS & $13(37)$ \\
\hline Malabsorption & $10(29)$ \\
\hline Fistula & $4(11)$ \\
\hline Obstruction & $3(9)$ \\
\hline Severe malnutrition prior to surgery & $1(3)$ \\
\hline Other & $4(11)$ \\
\hline
\end{tabular}


Fig. 4. QoL follow-up.

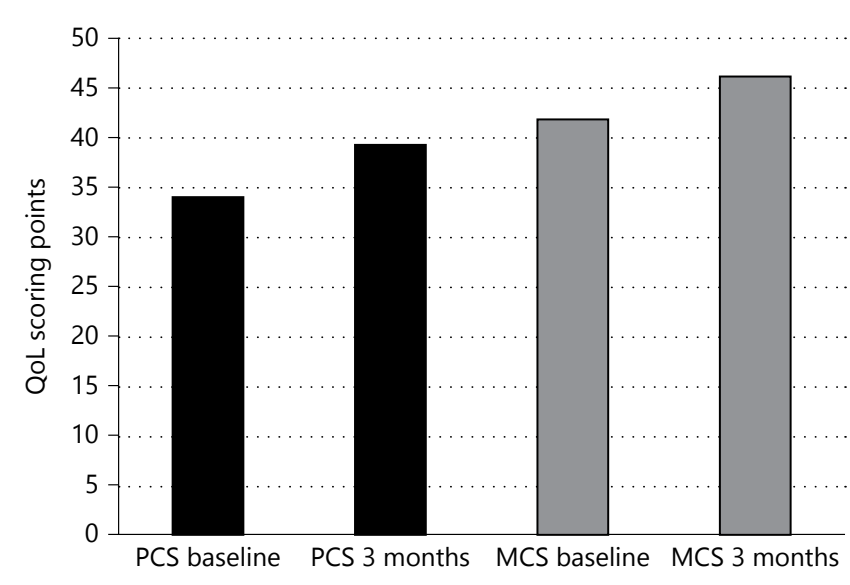

Fig. 5. HPN-related complaints.

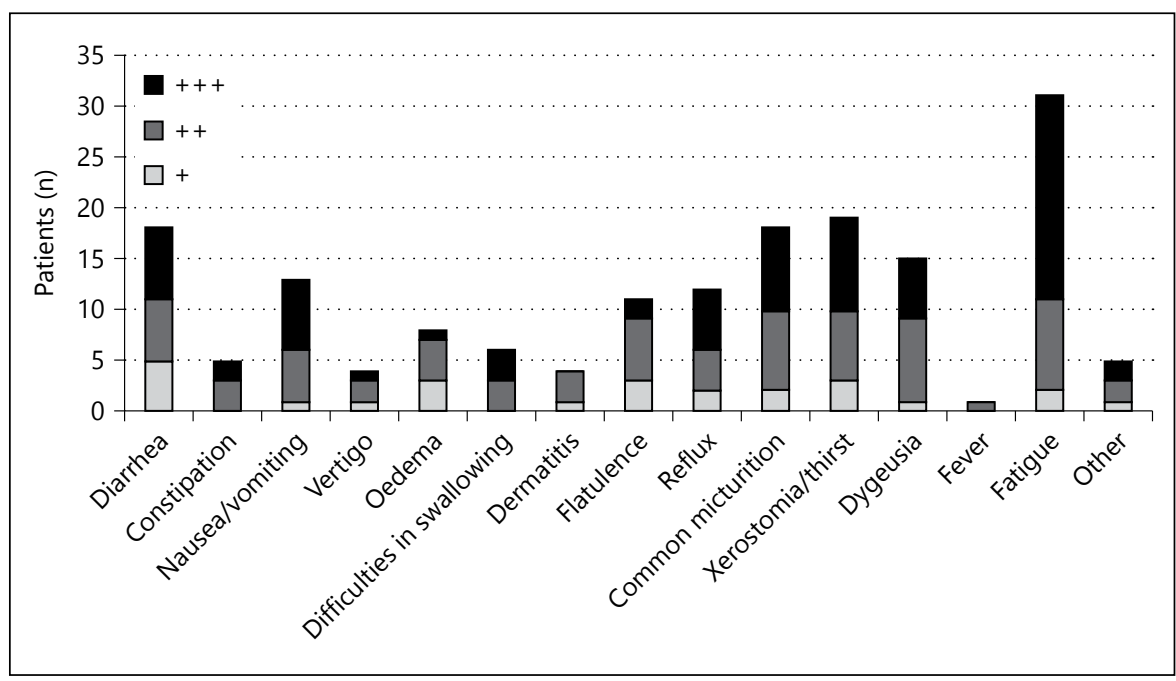

disturbances because of noise generated by the mechanical infusion pump were mentioned 8 times $(\mathrm{n}=8,24 \%)$. Fear of further health complications $(\mathrm{n}=5,15 \%)$ and problems in partnership/family life $(\mathrm{n}=3,9 \%)$ were also listed. The HPN-related complaints are shown in the figure 5. The complaint cited most often was by far fatigue ( $\mathrm{n}=31,94 \%$ ). Rarely occurring complaints were pains, spasms, difficulty in controlling blood sugar level, night sweats and post-prandial health problems.

\section{Discussion}

The present prospective study is the first detailed analysis of HPN patients in Switzerland. We consider this survey as representative for the population of HPN patients in our country. Of the $41 \mathrm{HPN}$ patients identified, 33 were included. The University Hospital of Berne provided 36\% of the recruited patients, far more than any other site, which may be due to the long lasting clinical experience with this population. HPN is prescribed not only by important referral centers, but also by general practitioners taking care of these patients. There are no regular followups, aspects or guidelines for HPN prescriptions and care in Switzerland.

The age and gender distribution in our study are comparable to those of other surveys, which also showed a much higher rate of female HPN patients [4, 13-15]. This is influenced by the underlying diseases, for example, post-radiation enteritis occurring in patients with gynecological malignancies. SBS may also be more likely to occur in women due to the shorter length of their small 
intestine [16]. A final reason is the increasing number of bariatric patients in Switzerland, of which $75 \%$ are women [17].

Compared to other studies, the mean duration of HPN in our cohort is slightly lower (mean 3.44 years), but with a broader range $(0.1-31$ years) $[10,18]$. There was also greater variation in our study due to the inclusion of a patient with Hirschsprung disease requiring HPN since birth and due to the inclusion of a patient with SBS since more than 30 years, corresponding to almost 9 times the mean duration of HPN.

Approximately, half of our patients have had at least one CVC replacement during HPN treatment. The complication mentioned most often was CVC infection. This corresponds with the results of previous studies: $28.6 \%$ had at least one catheter sepsis and $50 \%$ had at least one catheter change. CVC infection rate ranged between 0.38 and 4.58 episodes per 1,000 catheter days [ 19 , 20]. To prevent infections and other catheter-related complications, good clinical practice and an experienced multidisciplinary team are mandatory [21]. Six patients (18\%) needed a catheter replacement because of infectious complications in the 3 months of follow-up. This rate has to be interpreted with caution, because of the short follow-up period and because many patients just had started with the HPN therapy. The procedure in case of suspected CVC-related infection and subsequent removal is managed in a similar way throughout Switzerland. When a catheter infection is suspected, peripheral blood culture samples and culture samples from each catheter lumen are taken. If there is a definitive sign of local infection, for example, purulent secretion at the exit site, or a catheter-induced sepsis, the CVC is removed immediately and an antibiotic therapy is started [22].

In a European multicenter study, Van Gossum et al. [4] showed almost the same distribution of underlying diseases (cancer $42 \%$, Crohn's disease $15 \%$, vascular diseases 13\%). Recently, the ESPEN HPN-Chronic Intestinal Failure Special Interest Group (European Society of Clinical Nutrition and Metabolism) created a survey to describe the use of HPN in post-bariatric surgery, including the indications and outcome. An interesting finding in our study is that 4 patients (nearly 15\%) of our study population need HPN after bariatric surgery. Patients undergoing malabsorptive procedures are at risk of developing nutritional deficiencies and protein-energy malnutrition (PEM). A small number of patients will develop PEM and will therefore require HPN months to years after bariatric surgery. Bariatric surgery has increased significant- ly in the last years in Switzerland, with 750 bariatric interventions in 2001 compared with 4,000 in 2013 [17]. Not much is known about this group of patients requiring HPN to compensate for complications or side effects after bariatric surgery $[23,24]$. Given the proportion of morbidly obese people in the Swiss population (in 2012: 11\% men and 9\% women) [25], post-bariatric PEM will be an emerging problem in the future.

On the other hand, other indications have dramatically decreased, noticeably, the $4 \%$ proportion of AIDS patients in earlier years, most of who died of wasting syndromes due to the lack of effective therapies $[4,26]$. New treatments, better outcome and prevention strategies have increased survival and decreased the need for HPN in such patients [27]. This was also reflected in the fact that there were no AIDS patients in the current study. In the study of Van Gossum et al. [4], cancer was in first place (39\%) at almost the same rate like in our survey. The proportion of HPN patients with oncological tumors has increased over the past years in Europe [28]. Previous Swiss data about the indications for home artificial nutrition demonstrated that neoplasms were the most frequent underlying disease (51\%), diseases of the digestive system on the second place $(10.4 \%)$ and diseases of the nervous system in third place (9.6\%) [8]. In our study, there were no patients with underlying neurological disorders. This is probably due to the fact that patients with neurological disorders can mostly be treated with enteral nutrition, including PEG [29]. Another important fact is the change in official recommendations for HPN over the last years, repositioning HPN as a palliative treatment in cancer patients because of the relatively small proportion of $\mathrm{PN}$ associated comorbidity risk factors [14, 30]. Regarding the indications for HPN, we found that SBS is the most frequent indication (37\%), consistent with other published data [31, 32].

Our investigation also focused on the QoL of HPN patients. Both physical and mental QoL, assessed by the non-disease-specific SF-36v2, improved over time in our patients. Many other studies determined the QoL using the Karnofsky score, the SF-36 or a new specific questionnaire for HPN patients. Overall, patients with HPN have poorer QoL than healthy people or patients with chronic illnesses [13, 19, 32-34]. An earlier study with only 13 participants showed that none of those HPN patients had a regular employment, but $46 \%$ did most of the housekeeping [35]. Our data showed that $85 \%$ of patients had no employment, and only $39 \%$ were able to do the housekeeping on their own. 
The additional subjective definition of physical performance and mental health status were also investigated by Winkler et al. [36], who achieved greater insight into the lives of HPN patients and their definition of QoL. In our study, most of the patients reported that they were 'very active' or 'active' before disease, comparable with healthy individuals. In the immediate period before starting HPN, most of the patients felt very tired, adynamic and 'not active'. Fortunately, after initiation of HPN, the performance level increased after weeks in most patients, showing the positive effects of HPN on well-being, body function and performance levels. The results concerning mental health were similar, with 'poor' mental health reported before starting HPN and 'good' mental health after initiation of HPN.

We conclude that our population profits by HPN from a better health status. Other studies confirm our findings showing improved QoL with HPN in both patients with benign and malignant underlying disease [37-39].

Restricted travel options, limited social interaction and activities as well as loss of independence were the most frequent complaints related to HPN. Other studies showed similar results, mostly evaluated in surveys: the lack of freedom, being dependent and limitations in social life [40, 41]. Moreover, Huisman-de Waal et al. [42] showed social and somatic impacts of HPN therapy on daily life, which are comparable with our data (e.g. fatigue, xerostomia, diarrhea; fig. 5).

\section{Strengths and Limitations of the Study}

This prospective study provides the first detailed analysis of HPN patients in Switzerland. It is intended to regularly follow-up (in longer time periods) this cohort. The presented data will form the basis for a national HPN registry, which is needed to evaluate the specific situation of this cohort in the national (healthcare) context.

The fact that the follow-up of the study was only 3 months, and 3 patients had just started HPN before inclusion in the study is a limitation. The short duration was chosen to minimize patients lost to follow-up. Second, it was difficult for most patients during the survey to distinguish between HPN-related side effects and side effects due to underlying disease, and it was very challenging to determine whether improved or worsened QoL was caused by the underlying disease or is a direct consequence of the HPN. A disadvantage of our study is that we do not have the information about how many patients were eligible for the present study, but did not give their consent to the physicians in charge.

\section{Conclusion}

We conclude that $\mathrm{PN}$ is life saving and shows physical and mental benefit for HPN patients, but there are also negative implications for QoL, especially in terms of social and emotional aspects and loss of autonomy. The number of patients after bariatric surgery seems to increase steadily, and $\mathrm{PN}$ is effective in this population. This first prospective analysis of Swiss HPN patients, focusing on anthropometric parameters and QoL, shows major improvements over the 3 months study period. These patients should be monitored over the long term to evaluate and compare outcomes and the impact of HPN on the underlying disease and $\mathrm{HPN}$-associated complications. A national registry is a prerequisite for international comparability and benchmarking and can help to improve treatment quality and safety as well as to define best practices of HPN.

\section{Acknowledgments}

We would like to thank the SVK, particularly Mrs. N. Wagener and all the participating physicians for their contributions, namely: Dr. P. Coti (Lausanne), PD Dr. L. Genton (Geneva), Dr. J. Ravel (Geneva), Dr. R. Kehtari (Neuchâtel), Prof. R. Meier (Liestal), Dr. R. Villiger (Langnau), Dr. R. Hauser (Zurich), Dr. N. Ossola (Mendrisio), Dr. T. Wieland (Chur), Dr. A. Mayer (St. Gallen), PD Dr. F. Strasser (St. Gallen), Dr. S. Siegrist (St. Gallen), Dr. C. Krieger (St. Gallen). We would also like to thank the participating homecare providers for their cooperation.

\section{Statement of Authorship}

The authors declare that they have written the text themselves, and the work described herein is their own, unless otherwise acknowledged in the text. All sentences or passages quoted in this paper from other people's work have been specifically acknowledged by clear cross-referencing to author, work and page(s). All authors have participated sufficiently, intellectually or practically, in the present work and take public responsibility for the content of the article.

\section{Disclosure Statement}

None of the authors has a conflict of interest to declare.

\section{Funding Source}

The study was performed as part of a PhD thesis supported by a grant from the University of Basel FO119900 (Department of Clinical Pharmacy). 


\section{References}

1 Fish J, Steiger E, Seidner DL: Recent developments in home total parenteral nutrition. Curr Gastroenterol Rep 2000;2:327-330.

2 Seys P, Tadmouri A, Senesse P, Radji A, Rotarski M, Balian A, et al: Home parenteral nutrition in elderly patients with cancer: an observational prospective study. Bull Cancer 2014;101:243-249.

3 Marshall JK, Gadowsky SL, Childs A, Armstrong D: Economic analysis of home vs hospital-based parenteral nutrition in Ontario, Canada. JPEN J Parenter Enteral Nutr 2005; 29:266-269.

4 Van Gossum A, Bakker H, De Francesco A, Ladefoged K, Leon-Sanz M, Messing B, et al: Home parenteral nutrition in adults: a multicentre survey in Europe in 1993. Clin Nutr 1996;15:53-59.

5 Ugur A, Marashdeh BH, Gottschalck I, Brøbech Mortensen P, Staun M, Bekker Jeppesen P: Home parenteral nutrition in Denmark in the period from 1996 to 2001. Scand J Gastroenterol 2006;41:401-407.

6 Santarpia L, Pagano MC, Pasanisi F, Contaldo F: Home artificial nutrition: an update seven years after the regional regulation. Clin Nutr 2014;33:872-878.

7 Bakker H, Bozzetti F, Staun M, Leon-Sanz M, Hebuterne X, et al: Home parenteral nutrition in adults: a European multicentre survey in 1997. ESPEN-home artificial nutrition working group. Clin Nutr 1999;18:135-140.

8 Shaw SA, Rühlin M, Wagener N, Stanga Z, Meier R, Ballmer PE: Home artificial nutrition in Switzerland: an epidemiological survey from 2005 to 2009. Ann Nutr Metab 2013; 62:207-213.

9 Wanten G, Calder PC, Forbes A: Managing adult patients who need home parenteral nutrition. BMJ 2011;342:d1447.

10 Huisman-de Waal G, Naber T, Schoonhoven L, Persoon A, Sauerwein H, Van Achterberg T: Problems experienced by patients receiving parenteral nutrition at home: results of an open interview study. JPEN J Parenter Enteral Nutr 2006;30:215-221.

11 Ware JE, Snow KK, Kosinski M, Gandek B: SF-36 ${ }^{\circledR}$ Health Survey: Manual and Interpretation Guide. Boston, New England Medical Center, The Health Institute, 1993.

12 Bullinger M: Measuring health related quality of life. An international perspective. Adv Exp Med Biol 2003;528:113-122.

13 Pironi L, Baxter JP, Lauro A, Guidetti M, Agostini F, Zanfi C, et al: Assessment of quality of life on home parenteral nutrition and after intestinal transplantation using treatment-specific questionnaires. Am J Transplant 2012;12(suppl 4):S60-S66.

14 Hallum NS, Tan LB, Baxter JP, McKee RF: Home parenteral nutrition: outcome and seven year prospective follow up in a nationwide adult population. e-SPEN J 2012;7:e30-e34.

15 Moreno JM, Planas M, de Cos AI, Virgili N, Gómez-Enterría P, Ordóñez J, et al: The year
2003 national registry of home-based parenteral nutrition. Nutr Hosp 2006;21:127-131.

16 Nightingale JM, Lennard-Jones JE, Gertner DJ, Wood SR, Bartram CI: Colonic preservation reduces need for parenteral therapy, increases incidence of renal stones, but does not change high prevalence of gall stones in patients with a short bowel. Gut 1992;33:1493-1497.

17 Bauknecht F: Swiss Society for the Study of Morbid Obesity and Metabolic Disorders. SMOB, 2014. http://www.smob.ch/pdf/ Bariatrie\%20CH\%202001-2013\%20Auswer tungen\%20Website.pdf.

18 Gillanders L, Angstmann K, Ball P, O’Callaghan $\mathrm{M}$, Thomson A, Wong T, et al: A prospective study of catheter-related complications in HPN patients. Clin Nutr 2012;31:30-34.

19 Raman M, Gramlich L, Whittaker S, Allard JP: Canadian home total parenteral nutrition registry: preliminary data on the patient population. Can J Gastroenterol 2007;21:643-648.

20 Dreesen M, Foulon V, Spriet I, Goossens GA, Hiele M, De Pourcq L, et al: Epidemiology of catheter-related infections in adult patients receiving home parenteral nutrition: a systematic review. Clin Nutr 2013;32:16-26.

21 Dibb M, Teubner A, Theis V, Shaffer J, Lal S: Review article: the management of long-term parenteral nutrition. Aliment Pharmacol Ther 2013;37:587-603.

22 Jauch KW, Schregel W, Stanga Z, Bischoff SC, Brass P, Hartl W, et al: Access technique and its problems in parenteral nutrition - guidelines on parenteral nutrition, chapter 9 . Ger Med Sci 2009;7:Doc19.

23 Abdal Raheem S, Deen OJ, Corrigan ML, Parekh N, Quintini C, Steiger E, et al: Bariatric surgery complications leading to small bowel transplant: a report of 4 cases. JPEN J Parenter Enteral Nutr 2014;38:513-517.

24 Schiavone PA, Piccolo K, Compher C: Application of the A.S.P.E.N. clinical guideline for nutrition support of hospitalized adult patients with obesity: a case study of home parenteral nutrition. Nutr Clin Pract 2014;29:73-77.

25 Bundesamt für Statistik BFS: Schweizerische Gesundheitsbefragung, 2012 Übergewicht und Adipositas. Neuchâtel, Espace de l'Europe 10, 2014. http://www.bfs.admin.ch/ bfs/portal/de/index/news/publikationen. html?publicationID $=5806$.

26 Howard L, Heaphey L, Fleming CR, Lininger L, Steiger E: Four years of North American registry home parenteral nutrition outcome data and their implications for patient management. JPEN J Parenter Enteral Nutr 1991;15:384-393.

27 Ireton-Jones C, DeLegge M: Home parenteral nutrition registry: a five-year retrospective evaluation of outcomes of patients receiving home parenteral nutrition support. Nutrition 2005;21:156-160.

28 Mühlebach S: Heimparenterale ernährung internationale entwicklungen und langzeiterfahrungen aus der schweiz. Aktuel Ernaehr Med 2002;27:425-430.
29 Pironi L, Candusso M, Biondo A, Bosco A Castaldi P, Contaldo F, et al: Prevalence of home artificial nutrition in Italy in 2005: a survey by the Italian society for parenteral and enteral nutrition (SINPE). Clin Nutr 2007;26:123-132.

30 Cotogni P, Pittiruti M, Barbero C, Monge T, Palmo A, Boggio Bertinet D: Catheter-related complications in cancer patients on home parenteral nutrition: a prospective study of over 51,000 catheter days. JPEN J Parenter Enteral Nutr 2013;37:375-383.

31 Wanden Berghe C, Gómez Candela C, Chicharro L, Cuerda C, Martínez Faedo C, Virgili $\mathrm{N}$, et al: Home parenteral nutrition registry in Spain for the year 2010: NADYA-SENPE group. Nutr Hosp 2011;26:1277-1282.

32 Baxter JP, Fayers PM, McKinlay AW: The clinical and psychometric validation of a questionnaire to assess the quality of life of adult patients treated with long-term parenteral nutrition. JPEN J Parenter Enteral Nutr 2010;34:131-142.

33 Wong C, Lucas B, Wood D: Patients' experiences with home parenteral nutrition: a literature review. Eur J Hosp Pharm 2015;22:51-55.

34 Baxter JP, Fayers PM, McKinlay AW: A review of the quality of life of adult patients treated with long-term parenteral nutrition. Clin Nutr 2006;25:543-553.

35 Ladefoged K: Quality of life in patients on permanent home parenteral nutrition. JPEN J Parenter Enteral Nutr 1981;5:132-137.

36 Winkler MF, Hagan E, Wetle T, Smith C, Maillet JO, Touger-Decker R: An exploration of quality of life and the experience of living with home parenteral nutrition. JPEN J Parenter Enteral Nutr 2010;34:395-407.

37 Cozzaglio L, Balzola F, Cosentino F, DeCicco M, Fellagara P, Gaggiotti G, et al: Outcome of cancer patients receiving home parenteral nutrition. Italian society of parenteral and enteral nutrition (S.I.N.P.E.). JPEN J Parenter Enteral Nutr 1997;21:339-342.

38 Richards DM, Deeks JJ, Sheldon TA, Shaffer JL: Home parenteral nutrition: a systematic review. Health Technol Assess 1997;1:i-iii, 1-59.

39 Culine S, Chambrier C, Tadmouri A, Senesse P, Seys P, Radji A, et al: Home parenteral nutrition improves quality of life and nutritional status in patients with cancer: a French observational multicentre study. Support Care Cancer 2014;22:1867-1874.

40 Persoon A, Huisman-de Waal G, Naber TA Schoonhoven L, Tas T, Sauerwein H, et al: Impact of long-term HPN on daily life in adults. Clin Nutr 2005;24:304-313.

41 Winkler MF, Smith CE: Clinical, social, and economic impacts of home parenteral nutrition dependence in short bowel syndrome. JPEN J Parenter Enteral Nutr 2014;38(suppl 1):32S-37S

42 Huisman-de Waal G, Schoonhoven L, Jansen J, Wanten G, van Achterberg T: The impact of home parenteral nutrition on daily life - a review. Clin Nutr 2007;26:275-288. 